\title{
Effects of Laser Pulse Numbers on Surface Biocompatibility of Titanium for Implant Fabrication
}

\author{
Mitra Radmanesh, Amirkianoosh Kiani \\ Department of Mechanical Engineering, University of New Brunswick, Fredericton, Canada \\ Email: a.kiani@unb.ca
}

Received 2 June 2015; accepted 5 July 2015; published 9 July 2015

Copyright (C) 2015 by authors and Scientific Research Publishing Inc. This work is licensed under the Creative Commons Attribution International License (CC BY). http://creativecommons.org/licenses/by/4.0/

(c) (i) Open Access

\begin{abstract}
Generally, materials with high biocompatibility are more appropriate for bone and tissue transplant applications, due to their higher effectiveness in the healing process and infection problems. This study presents the effects of laser surface texturing on the surface topography properties, roughness, and wettability of thin titanium sheets, which consequently enhance the biocompatibility of this material. Creating line patterns across the surfaces, the titanium samples are prepared using variety of laser parameters. The apatite inducing ability of each sample is tested through the use of simulated body fluid (SBF). The final biocompatibility level of titanium samples is analyzed through wettability, surface angle measurements, and average surface temperature profile. Overall, the effects of laser parameter, pulse numbers, upon the biocompatibility of titanium are thoroughly examined, with results indicating that a scanning speed of $100 \mu \mathrm{m} / \mathrm{ms}$ results in desirable bone type apatite inducing abilities across the surface of treated titanium sheets.
\end{abstract}

\section{Keywords}

Laser Surface Micro Texturing, Titanium, Implant, Biocompatability, Hydroxyapatite Deposition

\section{Introduction}

The fields of biomedical and tissue engineering have been expanded immensely in previous decades. Development of new technologies to enhance the biocompatibility of a wider range of materials in a short and efficient manner is one of the important challenges in these fields. Biocompatibility issues are notably improved by the introduction of laser surface micro/nano texturing techniques, and through the fast, precise, and reliable operation of these methods. Micro/sub-micro treated titanium surfaces are popularly used in scaffold systems in bone 
and tissue implant applications. High level of biocompatibility of titanium, is essential in the implant fabrication for avoiding any infections and immune system rejection while providing the required structural properties for the implant [1] [2]. An effective way of enhancing the biocompatibility of a material is to alter its surface properties, such as surface structure and topography [3]. The surface topography properties of a material, espeically the roughness properties, are very influential on the apatite inducing ability of bone type apatite to surfaces. Increasing the apatite deposition rate across the surface of a material increases the biocompatibility of that material as well [4] [5]. The purpose of this paper is to investigate the effects of laser surface texturing on enhancing the biocompatibility of titanium. The surface topography properties of the titanium samples are altered through a range of laser process parameters, and effects of pulse rate on surface structure, roughness, and wettability of titanium are examined. The biocompatibility of the processed samples is then examined by performing wettability analysis and through the use of simulated body fluid (SBF) and the comparison of multiple samples. The essential purpose of this research is to study the behavior and effects of laser scanning parameters. Due to the fact that these parameters vary in a wide range, understanding their effects on surface treatment and biocompatibility could provide an efficient manner for utilizing the lasers for biomedical applications.

\section{Experimental Setup}

\subsection{Laser System \& Sample Preparation}

Thin sheets of titanium were chosen for conducting the experiment. The samples were initially irradiated with nanosecond pulses to create the predetermined line patterns on their surfaces with specific laser parameters. The experiment was completed using a pulse repetition rate (frequency) of $25 \mathrm{kHz}$; while the scanning speed was set to $100 \mu \mathrm{m} / \mathrm{ms}, 300 \mu \mathrm{m} / \mathrm{ms}$, and $500 \mu \mathrm{m} / \mathrm{ms}$, using the $\mathrm{EZCAD}^{\odot}$ software. The laser power throughout the experiment was measured to be $7 \mathrm{~W}$, which was calculated using a power-meter prior to processing of the samples. The following Table 1 displays the experiment design parameters in more detail.

\subsection{SBF Processing \& in Vitro Assessment}

In the next phase of the study, the surface-treated samples were soaked in simulated body fluid, in order to assess their altered biocompatibility. To prepare simulated body fluid, a strict step-by-step method had to be followed. Since SBF is supersaturated with respect to apatite, failing to follow the preparation procedure accurately could lead to precipitation of apatite in the solution [6]. Given the exact amount of the required ingredients, the mixture of SBF must be conceived at controlling conditions, in terms of temperature and $\mathrm{pH}$ levels. After dissolving the exact amount of the given chemicals in order, using Tris and Hydrochloric acid, the $\mathrm{pH}$ level of the solution was controlled to be in the range of $7.40-7.45$ at a temperature of 36.5 degrees Celsius exactly. It was essential to ensure that the $\mathrm{pH}$ of the solution did not increase beyond 7.45 at any point during the preparation. The solution was finalized once the $\mathrm{pH}$ level of the solution was adjusted at 7.40 at a temperature of 36.5 degrees Celsius exactly [6].

Upon conceiving the simulated body fluid, the surface treated titanium sheets were soaked in the fluid for a period of three days. The soaked samples were then examined using scanning electronic microcopy and by performing surface angle measurement analysis across their surfaces. The obtained results are presented and discussed in the following sections.

\section{Results \& Discussion}

\subsection{Structure of Line Patterns across the Surface}

Surface topography properties of materials are directly influential on their apatite-inducing ability. Essential laser

Table 1. Laser parameters used in surface treating of samples.

\begin{tabular}{cccc}
\hline & \multicolumn{3}{c}{ Laser Parameters } \\
\hline Frequency $(\mathrm{KHz})$ & Power $(\mathrm{W})$ & Scanning Speed $(\mu \mathrm{m} / \mathrm{ms})$ & Number of Pulses \\
\hline \multirow{2}{*}{25} & & 100 & 5 \\
& 7 & 300 & 1.7 \\
& & 500 & 1 \\
\hline
\end{tabular}


parameters such as pulse numbers can effect these properties greatly in many ways. Generally, upon laser irradiation, the surface structure and patterns of material change result in increased roughness and surface oxidation. Surface roughness is directly related to the availability of the exposed surface of a material. The exposed surface area of a work piece is more readily available upon an increase of surface roughness, which in return enhances the apatite-inducing ability and cell adhesion rate of a material. A larger surface area is exposed, and a larger number of cells can interact with surface; hence, a larger rate of apatite inducing can take place, which is the essential goal of this experiment [5] [7] [8]. Further, laser treatment of the titanium results in the creation of thin layers of titanium oxide (titania), through an increase in surface temperature of material up to the oxidation temperature [9] [10]. The created layer of titania across the surface of titanium, results in a cushion-type layer available for cells to more easily attach across the surface; this therefore results in a higher apatite perception across the treated surface of the titanium sheets [11]. An increase in the oxidation of the surfaces is directly effective on increasing the wettability of surface materials, which is also directly related to surface roughness. This leads to an increase in the apatite-inducing ability of the material, and greatly improves the biocompatibility of the implant surfaces [9]. This effect is further analyzed through the obtained results of this study.

\subsection{Surface Angle Measurements; Wettability Analysis}

Surface roughness and wettability are directly related. By increasing the surface roughness and oxidation of a material, the wettability of the material increases as well, which is an indicator of the ability of that particular surface to absorb more. This results in a porous effect upon the surface of the material, and is very effective in enhancing the apatite-inducing ability of the material [11] [12]. Upon imposing the line patterns on the surfaces of titanium sheets, their wettability is analyzed. Figure 1 displays the average surface angles measured for samples prepared with scanning speeds of $100 \mu \mathrm{m} / \mathrm{ms}, 300 \mu \mathrm{m} / \mathrm{ms}$, and $500 \mu \mathrm{m} / \mathrm{ms}$.

Scanning speed directly influences the number of laser pulses. An increase in the scanning speed decreases the number of pulses irradiated upon the surface of the samples; hence, a scanning speed of $500 \mu \mathrm{m} / \mathrm{ms}$ has the lowest pulse numbers (Table 1 ).

As shown in Figure 1, the surface angle of the samples increases as the scanning speed increases. This means that at lower pulse numbers there is a larger contact angle between the surfaces of titanium and water. The increase in the contact angle is an indicator that with a decrease in pulse number, the surface of the titanium is modified from hydrophilic to hydrophobic. This is due to the fact that lower pulse numbers have much less effect on the surface roughness and oxidation of material, since there is a shorter period of interaction between the laser and the material's surface [11] [13]. The hydrophilic properties of a surface cause the liquid to penetrate deeper into the surface; consequently, a smaller contact angle results, which is a strong indicator of improvement in wettability of that material. Having a high wettability produces a porous temple for the cells to easily absorb and attach to the surface of titanium, and thereby enhance its biocompatibility. Looking at Figure 1, it is easy to see that the wettability of the sample prepared with a scanning speed of $100 \mu \mathrm{m} / \mathrm{ms}$ (pulse rate: 5) has the highest wettability due to the largest number of pulses. As mentioned, the higher number of pulses notably

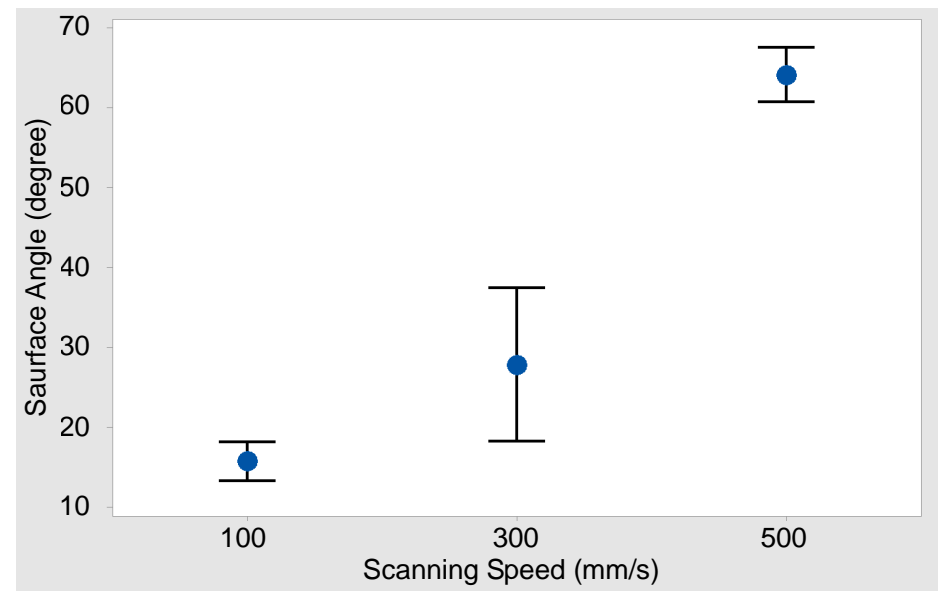

Figure 1. Average surface contact angle - wettability analysis. 
increases the roughness and oxidation of the titanium surface, and results in a better porous effect across the surface [7] [11] [14].

\subsection{SBF Soaking of Surface Treated Titanium Sheets}

To fully examine the effects of pulse number on roughness, oxidation, and wettability of titanium sheets, and their biocompatibility, the samples were soaked in SBF for three days. Figure 2 and Figure 3 display the SEM micrographs of the apatite-inducing ability of samples prepared by power $7 \mathrm{~W}$ and scanning speeds of 100 $\mu \mathrm{m} / \mathrm{ms}, 300 \mu \mathrm{m} / \mathrm{ms}$, and $500 \mu \mathrm{m} / \mathrm{ms}$.

As it can be seen from Figure 2, the imposed line patterns on the surface of titanium sheets has made the surface rougher, and as a result, a thicker bone-type apatite layer precipitated upon each surface. The deposition of these layers is an indicator of the apatite-inducing ability of the processed samples, and hence a higher biocompatibility. The thicker the bone-apatite layer deposition is, the higher the apatite-inducing ability is, and therefore the higher the biocompatibility of the titanium sheets is.

Figure 3 displays a closer view of the titanium samples soaked in SBF. Comparing the three samples in this figure, it can be seen that the bone like apatite layer across Figure 3(A) is more developed compared to the other two samples. As mentioned, a lower scanning speed results in a higher number of pulses. In this sample, more energy was transferred from laser to the surface of titanium, and the roughness of the surface is highly altered compared to other two cases. Therefore, a better apatite-inducing ability results in this sample. Similarly, comparing Figure 2(B) and Figure 2(C), the apatite-inducing ability of Figure 3(B) is better than the previous sample, since it has a larger number of pulses in comparison.

Further, as stated, the heat rise is larger with more number of pulses; therefore, the temperature of a larger area of the surface would reach the oxidation level. This in return will turn a larger amount of titanium into titanium oxide. As stated, titanium oxide increases the wettability of the surface material. This is in agreement with the results obtained from the wettability analysis of the titanium sheets. Sample A) with the greatest number of pulses, had the lowest contact angle, and hence the highest wettability. As stated, when the wettability of a material is increased, a higher surface energy exists across the surface of that material, and therefore a better

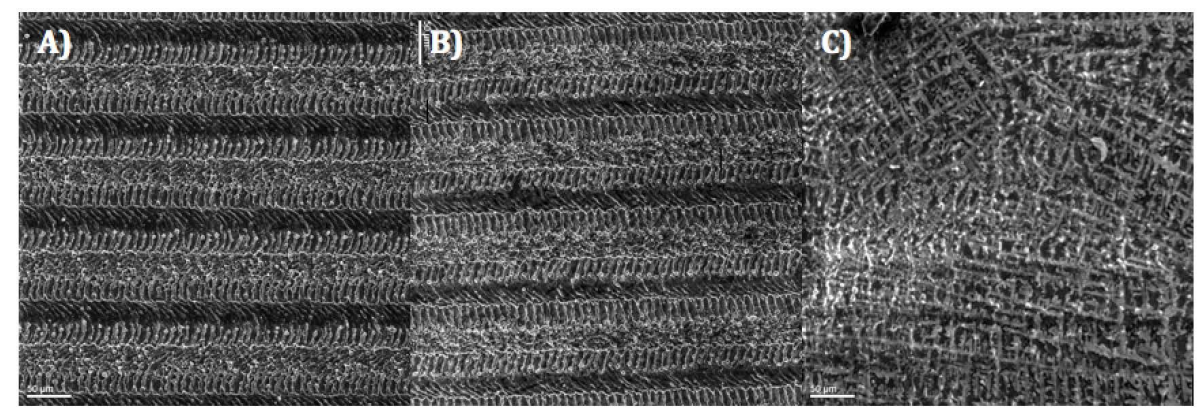

Figure 2. A) $100 \mu \mathrm{m} / \mathrm{ms}$; B) $300 \mu \mathrm{m} / \mathrm{ms}$; C) $500 \mu \mathrm{m} / \mathrm{ms}$; Power: $7 \mathrm{~W}$, F: $25 \mathrm{KHz}$; Overview of the Roughness Level of each Scanning Speed and the According Apatite Deposition Layer.

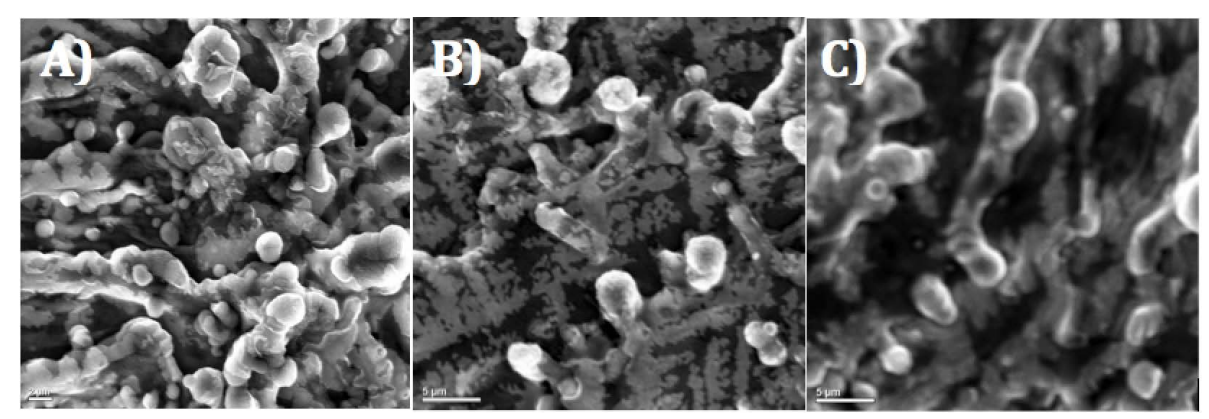

Figure 3. Zoomed SEM images of A) $100 \mu \mathrm{m} / \mathrm{ms}$; B) $300 \mu \mathrm{m} / \mathrm{ms}$; C) $500 \mu \mathrm{m} / \mathrm{ms}$; Power: 7 W, F: $25 \mathrm{KHz}$ 
meditation of bone-like apatite deposition can be achieved. Essentially, the higher apatite inducing ability provides a better cell attachment rate [11] [14] [15].

Figure 4 shows the magnified SEM image of the deposited ion-substituted carbonate apatite layer formed by SBF treatment. In this figure, the arrows refer to the bone-like apatite fully developed across the treated surface of the samples, much similar to the apatite layers in Figure 3.

\subsection{Temperature Profile Analysis of Treated Samples}

To verify the accuracy of the obtained observations, a thorough theoretical analysis of effects of laser parameters upon the surface roughness and wettability of the samples is conducted. In order to fully understand the degree of change in surface roughness and wettability of each sample, tracking the changes in the surface temperature across the samples due to number of pulses is an ideal approach. In this study, the one-dimensional heat conduction equation is assumed to be [16]:

$$
\frac{\partial T}{\partial t}=a \frac{\partial^{2} T}{\partial X^{2}}
$$

where $a$ is the thermal diffusivity coefficient.

In this analysis, the thermal diffusivity coefficient is calculated using the following relation [17] [18]:

$$
a=\frac{k}{\rho c_{p}} .
$$

where $k$ is the thermal conductivity coefficient, $\rho$ is the density, and $c_{p}$ is the specific heat of titanium.

To simplify the heat conduction equation, the profile of laser pulse is taken to be a rectangular profile with the step-like rise and fall; therefore the temperature profile for each laser pulse is obtained as:

$$
T(X, t)=k \sqrt{\frac{a}{\pi}} \int_{0}^{t_{p}} \frac{I_{a(\tau)}}{\sqrt{t-\tau}} \exp \left\{-\frac{X^{2}}{2 a(t-\tau)}\right\} \mathrm{d} \tau .
$$

Next the maximum temperature, occurring at the end of each laser pulse, is required to develop the surface temperature profile, which can be approximately calculated as [18] [19]:

$$
T\left(0, t_{p}\right)=T_{\max } k \sqrt{\frac{2 a}{\pi^{3} t_{p}}} \frac{4 K(1-R) P}{k \mathcal{F} d^{2}} .
$$

where $t_{p}$ is the pulse duration, $k$ is the thermal conductivity coefficient, $P$ is the average power, $\mathcal{F}$ is the frequency, $d$ is the laser spot diameter, $R$ is the reflection coefficient, and $K$ is the residual energy coefficient. The reflection coefficient is calculated to be 0.56 for wavelength of $1064 \mathrm{~nm}$, the residual energy coefficient

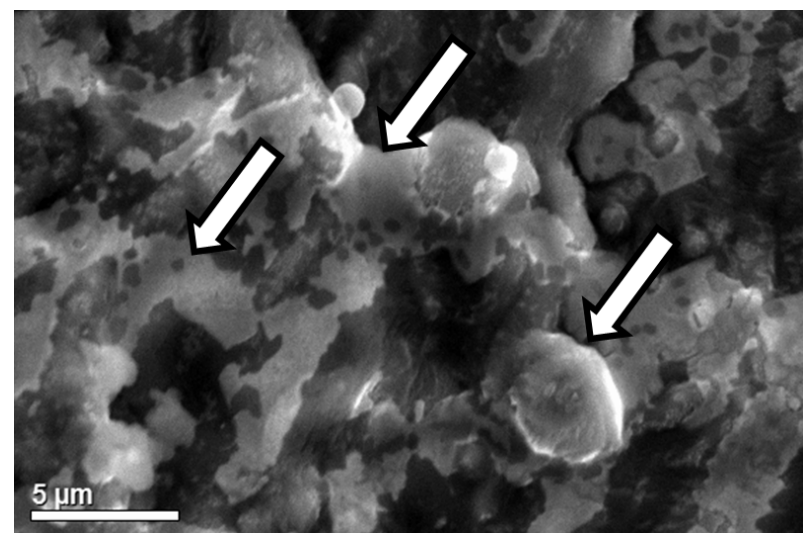

Figure 4. SEM image of deposited apatite layer after soaking in SBF; A closer magnification of the apatite developing across the surface of titanium. 
is taken as 0.45 , the thermal diffusivity coefficient is obtained 7.068exp-6, and laser spot diameter calculated as $30 \mu \mathrm{m}$.

Finally, the average temperature across the surface of the samples is obtained as [17] [19]:

$$
T_{n}=2 a \frac{\left[1-\frac{2}{3} \alpha\right]}{\left(1+\alpha^{2}\right)} \frac{T_{n}}{(1+\alpha)}\left[1+\frac{\alpha^{n}-\alpha}{n(1-\alpha)}\right] .
$$

where $T_{m}$ is the maximum temperature calculated using Equation (1), $n$ is the pulse numbers, and $\alpha$ is laser pulse which is given by $T_{\min }=\alpha T_{\max }$. Where $\alpha$ is the constant ratio for the previous maximum and the following minimum temperatures and equals to $\alpha=\left(t_{p} / t_{p p}\right)^{0.5}$

The presented analytical results of the study are associated with possible errors due to multiple assumptions made throughout the work. However, the discussed results accurately display the trend of temperatures, which is the most important aspect of the analysis. The temperature profile using power of $7 \mathrm{~W}$, Frequency of $25 \mathrm{KHz}$, for each of the cases of study is obtained by using a pulse number range of 1 pulse through 50 pulses. Figure 5 displays the temperature profile obtained in this study:

Figure 5 displays the average surface temperature for the samples using a power of $7 \mathrm{~W}$. As shown, the temperature rises with an increase in the number of pulses irradiated on the surface. The high temperature causes more ablation on the surface of the material, which in return increases the roughness and oxidation of the surface. The trend of temperature change is in very close agreement with the obtained observations in this study. At lower scanning speeds, a higher number of pulses are obtained, and therefore a higher surface temperature is achieved. Therefore, the sample prepared using a scanning speed of $100 \mu \mathrm{m} / \mathrm{ms}$ has higher roughness across the surface with a better wettability. Examining the apatite-inducing ability of this sample as discussed in Figure 2 shows this clearly [20].

\section{Conclusion}

Through this study, the effects of laser surface texturing on the apatite-inducing ability of titanium were investigated, using different scanning speeds (thus different pulse numbers), in preparation of line patterns across the surface of the samples. By increasing the surface roughness of the samples, the wettability of the samples increased, and hence a cushion-type temple was created that produced an effective environment for cell attachment to take place. The biocompatibility of the titanium sheets was thereby enhanced to be more applicable in bone and tissue transplant applications. The samples, after pre-defined line patterns on their surfaces were generated, were placed in simulated body fluid for three days before being tested for their biocompatibility. The immersed samples were then analyzed using SEM and wettability to evaluate the bone-type apatite-inducing ability on the surface of each set of samples. Also, the effects of pulse numbers on the surface roughness, wettability,

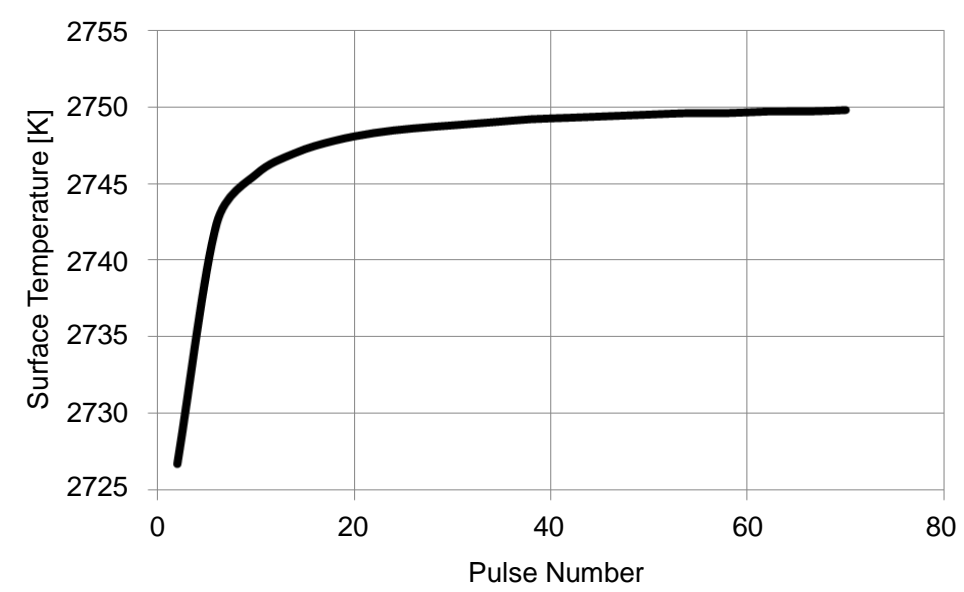

Figure 5. Average surface temperature profile based on a range of pulse numbers; Power $7 \mathrm{~W}$. 
and oxidation level of the material were compared. Upon completion of the analysis, it is observed that using lower scanning speeds results in higher pulse numbers; therefore, higher surface temperatures at the irradiated spots and larger roughness and oxidation levels are achieved. This leads to better apatite-inducing ability across the surface of the titanium sheets. Overall, the experimental observations are in close agreement with the analytical results.

\section{Acknowledgements}

This research is funded by the Natural Science and Engineering Research Council of Canada (NSERC) and the New Brunswick Innovation Foundation (NBIF).

\section{References}

[1] Hutmacher, D.W. (2000) Scaffolds in Tissue Engineering Bone and Cartilage. Biomaterials, 21, 2529-2543. http://dx.doi.org/10.1016/S0142-9612(00)00121-6

[2] Ahmmed, K.T., Ling, E.J.Y., Servio, P. and Kietzig, A.M. (2015) Introducing a New Optimization Tool for Femtosecond Laser-Induced Surface Texturing on Titanium, Stainless Steel, Aluminum and Copper. Optics and Lasers in Engineering, 66, 258-268. http://dx.doi.org/10.1016/j.optlaseng.2014.09.017

[3] Coelho, P.G., Granjeiro, J.M., Romanos, G.E., Suzuki, M., Silva, N.R., Cardaropoli, G., Thompson, V.P. and Lemons, J.E. (2009) Basic Research Methods and Current Trends of Dental Implant Surfaces. Journal of Biomedical Materials Research Part B: Applied Biomaterials, 88, 579-596. http://dx.doi.org/10.1002/jbm.b.31264

[4] Ulerich, J.P., Ionescu, L.C., Chen, J.B., Soboyejo, W.O. and Arnold, C.B. (2007) Modifications of Ti-6Al-4V Surfaces by Direct-Write Laser Machining of Linear Grooves. Proceedings of SPIE 6458, Photon Processing in Microelectronics and Photonics VI, 645819. http://dx.doi.org/10.1117/12.713964

[5] Erdoğan, M., Öktem, B., Kalaycioğlu, H., Yavaş, S., Mukhopadhyay, P.K., Eken, K., Ilday, F.Ö., et al. (2011) Texturing of Titanium (Ti6Al4V) Medical Implant Surfaces with MHz-Repetition-Rate Femtosecond and Picosecond YbDoped Fiber Lasers. Optics Express, 19, 10986-10996. http://dx.doi.org/10.1364/OE.19.010986

[6] Kokubo, T. and Takadama, H. (2006) How Useful Is SBF in Predicting in Vivo Bone Bioactivity? Biomaterials, 27, 2907-2915. http://dx.doi.org/10.1016/j.biomaterials.2006.01.017

[7] Mirhosseini, N., Crouse, P.L., Schmidth, M.J.J., Li, L. and Garrod, D. (2007) Laser Surface Micro-Texturing of Ti-6Al-4V Substrates for Improved Cell Integration. Applied Surface Science, 253, 7738-7743. http://dx.doi.org/10.1016/j.apsusc.2007.02.168

[8] van Tol, A.F., Tibballs, J.E., Gjerdet, N.R. and Ellison, P. (2013) Experimental Investigation of the Effect of Surface Roughness on Bone-Cement-Implant Shear Bond Strength. Journal of the Mechanical Behavior of Biomedical Materials, 28, 254-262. http://dx.doi.org/10.1016/j.jmbbm.2013.08.005

[9] Tavangar, A., Tan, B. and Venkatakrishnan, K. (2011) Synthesis of Bio-Functionalized Three-Dimensional Titania Nanofibrous Structures Using Femtosecond Laser Ablation. Acta Biomaterialia, 7, 2726-2732. http://dx.doi.org/10.1016/j.actbio.2011.02.020

[10] Wang, H.S., Liang, C.Y., Yang, Y. and Li, C.Y. (2010) Bioactivities of a Ti Surface Ablated with a Femtosecond Laser through SBF. Biomedical Materials, 5, Article ID: 054115. http://dx.doi.org/10.1088/1748-6041/5/5/054115

[11] Das, K., Balla, V.K., Bandyopadhyay, A. and Bose, S. (2008) Surface Modification of Laser-Processed Porous Titanium for Load-Bearing Implants. Scripta Materialia, 59, 822-825. http://dx.doi.org/10.1016/j.scriptamat.2008.06.018

[12] Kazemi, K. and Goldak, J.A. (2009) Numerical Simulation of Laser Full Penetration Welding. Computational Materials Science, 44, 841-849. http://dx.doi.org/10.1016/j.commatsci.2008.01.002

[13] De Aza, P.N., Fernandez-Pradas, J.M. and Serra, P. (2004) In Vitro Bioactivity of Laser Ablation Pseudowollastonite Coating. Biomaterials, 25, 1983-1990. http://dx.doi.org/10.1016/j.biomaterials.2003.08.036

[14] Nolte, S., Momma, C., Jacobs, H., Tünnermann, A., Chichkov, B.N., Wellegehausen, B. and Welling, H. (1997) Ablation of Metals by Ultrashort Laser Pulses. Journal of the Optical Society of America B, 14, 2716-2722. http://dx.doi.org/10.1364/JOSAB.14.002716

[15] Fasasi, A.Y., Mwenifumbo, S., Rahbar, N., Chen, J., Li, M., Beye, A.C., Arnold, C.B. and Soboyejo, W.O. (2009) Nano-Second UV Laser Processed Micro-Grooves on Ti6Al4V for Biomedical Applications. Materials Science and Engineering: C, 29, 5-13. http://dx.doi.org/10.1016/j.msec.2008.05.002

[16] Kiani, A., Venkatakrishnan, K., Tan, B. and Venkataramanan, V. (2011) Maskless Lithography Using Silicon Oxide Etch-Stop Layer Induced by Megahertz Repetition Femtosecond Laser Pulses. Optics Express, 19, 10834-10842. http://dx.doi.org/10.1364/OE.19.010834 
[17] Kiani, A., Venkatakrishnan, K. and Tan, B. (2010) Direct Laser Writing of Amorphous Silicon on Si-Substrate Induced by High Repetition Femtosecond Pulses. Journal of Applied Physics, 108, Article ID: 074907. http://dx.doi.org/10.1063/1.3493192

[18] Kuang, J.H., Hung, T.P., Lai, K., Hsu, C.M. and Lin, A.D. (2012) The Surface Absorption Coefficient of S304L Stainless Steel by Nd: YAG Micro-Pulse Laser. Advanced Materials Research, 472, 2531-2534. http://dx.doi.org/10.4028/www.scientific.net/AMR.472-475.2531

[19] Venkatakrishnan, K., Stanley, P., Sivakumar, N.R., Tan, B. and Lim, L.E.N. (2003) Effect of Scanning Resolution and Fluence Fluctuation on Femtosecond Laser Ablation of Thin Films. Applied Physics A, 77, 655-658. http://dx.doi.org/10.1007/s00339-002-1668-1

[20] Ramsden, J.J., Allen, D.M., Stephenson, D.J., Alcock, J.R., Peggs, G.N., Fuller, G. and Goch, G. (2007) The Design and Manufacture of Biomedical Surfaces. CIRP Annals-Manufacturing Technology, 56, 687-711. http://dx.doi.org/10.1016/j.cirp.2007.10.001 